\title{
Measuring the Balance Space Sensitivity in Vector Optimization*
}

\author{
Alejandro Balbás ${ }^{\dagger} a n d$ Pedro Jiménez Guerra ${ }^{\ddagger}$
}

\begin{abstract}
Recent literature has shown that the balance space approach may be a significant alternative to address several topics concerning vector optimization. Although this new look also leads to the efficient set and, consequently, is equivalent to the classical viewpoint, it yields new results and algorithms, as well as new economic interpretations, that may be very useful in theoretical frameworks and practical applications. The present paper focuses on the sensitivity of the balance set. We prove a general envelope theorem that yields the sensitivity with respect to any parameter considered in the problem. Furthermore, we provide a dual problem that characterizes the primal balance space and its sensitivity. Finally, we also give the implications of our results with respect to the sensitivity of the efficient set.
\end{abstract}

\section{Introduction}

Since the concepts of balance point and balance set were introduced in [6] for vector optimization problems, several authors have analyzed their significant properties and have developed some algorithms to compute them in practice (see [5], [6], [7], [8] and [9] for further details).

Mainly, this approach yields a very general alternative method in vector optimization because multiobjective problems may be deeply analyzed by means of their ideal points rather than scalarized problems. It is not necessary to seek appropriate weights to compute a balance point. Instead, one has to choose a direction of preferential deviations from the ideal point in order to reach an optimal point.

\footnotetext{
-We would like to thank Professor Efim A. Galperin for very helpful comments and suggestions.

†Universidad Carlos III. Departamento de Economía de la Empresa. C/ Madrid 126. 28903 Getafe, Madrid (Spain). balbas@emp.uc3m.es

¥U.N.E.D. Departamento de Matemáticas Fundamentales. C/ Senda del Rey s/n. 28040 Madrid (Spain). pjimenez@mat.uned.es
} 
Consequently, an interesting economic meaning is possible since the ideal point may be considered an adequate reference for the decision maker. Given an arbitrary balance point $b=\left(b_{1}, b_{2}, \ldots, b_{n}\right), b_{i}$ is the difference between the final level attained in the $i^{\text {th }}$ objective and its "ideal level", and thus, the decision maker can choose another balance point when these differences are not very successful. Furthermore, $b$ is proportional to the direction used to leave the ideal point and, therefore, each quotient $b_{i} / b_{j}$ provides the number of units lost in the $i^{\text {th }}$ objective per unit lost in the $j^{t h}$ one. When the problem is scalarized, the meaning of the weights is not so clear.

This nonscalarized procedure provides new algorithms which, as said above, are very general. When we are choosing a concrete direction to detect a balance point we are also choosing ratios of losses among the conflicting objectives.

As pointed out in [5], the set of Pareto solutions and the balance set are equivalent from a theoretical viewpoint, in the sense that there exists a simple relationship between both sets. Thus, balance set techniques also apply to study the Pareto solutions.

Both advantages, new nonscalarized algorithms and economic interpretations, justify the interest of extending the discussion in order to address another important issues of vector optimization. So, this paper focuses on duality and sensitivity since these topics still present many open questions when dealing with vector problems.

Regarding duality and sensitivity, scalar problems have many deep properties whose extension for vector problems is not straightforward. This fact is clearly pointed out in [10] and [15], where interesting, general and classical treatments are presented. Although significant advances have been achieved and useful developments have been provided from both theoretical and practical viewpoints, in general, the dual objective is given by multifunctions ${ }^{1}$ : and this situation makes it rather difficult to establish saddle point conditions or sensitivity results. In fact, statements are far more complicated than the corresponding results for scalar problems, and their practical applications present larger difficulties too. Linear vector problems are much easier but, as pointed out in [1] and [4], even this case becomes complex when using flexible criteria to choose an optimal solution in the efficient set. ${ }^{2}$

In order to overcome these difficulties the recent literature (see for instance $[2],[3],[11]$ or [12]) has developed new ideas and methods, and we will try to show here that the balance space approach may be an useful alternative and can broaden possible techniques. Most of the classical caveats disappear

${ }^{1}$ i.e., if $\lambda$ represents the dual objective and $y$ is a dual feasible element, then $\lambda(y)$ is not vector but a set of vectors.

${ }^{2}$ For instance, in order to guarantee that dual solutions measure the primal sensitivity with respect to the vector in the right side, one has to impose restrictive assumptions with regard to the weights used when composing an efficient solution as a linear corvex combination of extreme points. when dealing with the ideal point sensitivity ${ }^{3}$ (see for instance [16] for a complete analysis that holds in a very general framework) and, therefore, since the efficient set equals the ideal point plus the balance set (see [5]), the sensitivity of this set would allow us to obtain the primal sensitivity by adding two terms

The remainder of the paper is as follows. Section 2 presents the basic concepts, notations and hypotheses. Section 3 deals with the primal sensitivity. First of all we establish Theorem 1, that yields a scalar programming problem whose solution leads to the balance point proportional to the direction of preferential deviations. ${ }^{4}$ Later, we draw on the sensitivity of this scalar problem, along with the sensitivity of the ideal point, and prove a general envelope theorem (Theorem 2) that provides the sensitivity of a general vector problem with respect to any parameter considered in the problem. Theorem 2 gives the sensitivity by means of a far simpler analytic expression than those provided by previous literature (see for instance [2]). Accordingly; the sensitivity may be easily computed in practical applications. Corollary 3 presents the sensitivity with respect to the vector of the right side.

The duality theory for convex problems is addressed in Section 4. Theorem 1 allows us to introduce a dual problem for which several properties are proved. So, the dual objective is never greater than the primal one, the absence of duality gap may by stated, dual and primal solutions are characterized by saddle points and complementary slackness conditions and, finally. the dual problem is linear if so is the primal one. Furthermore, the dual solutions coincide with the Lagrange multipliers and, according to our general envelope theorem, provide the primal sensitivity. Theorem 6 might merit particular attention since it provides special saddle points and slackness conditions that are given by means of vector inequalities and, consequently, may be easily applied in practical applications. ${ }^{5}$

The last section concludes and summarizes the article.

\section{Preliminaries and Notations}

Let $k, l, m$ and $n$ be entire numbers, $W$ and $P$ subsets of $\mathbb{R}^{n}$ and $\mathbb{R}^{l}$ respectively, $d=\left(d_{1}, d_{2}, \ldots, d_{k}\right) \in \mathbb{R}^{k}$ with $d_{i} \geq 0$ for $i=1,2, \ldots, k$ and $d \neq 0$, and $f: W \longrightarrow \mathbb{R}^{k}$ and $g: W \times P \longrightarrow \mathbb{R}^{m}$ two vector functions whose real components will be denoted by $f_{i}, i=1,2, \ldots, k$, and $g_{j}, j=1,2, \ldots, m$, respectively.

\footnotetext{
${ }^{3}$ Consequently, if there are no conflicts among the objectives and all of them attain the optimal value at the same feasible point, then the main properties of the scalar programming may be extended without introducing more complex statements and stronger assumptions.

${ }^{4}$ As said above, the direction of preferential deviations may be easily interpreted because it provides ratios of losses in each objective.

${ }^{5}$ In fact, in the statement of Theorem 6 we will use the symbol $\leq$ instead of $\nsucceq$. However, previous literature often uses $\nsucceq$ unless the author deals with non-conflicting objectives.
} 
Let us focus on the following vector optimization problem

$$
\operatorname{Min} f(x) \quad\left\{\begin{array}{l}
x \in W \\
g(x, p) \leq 0
\end{array}\right.
$$

being $p \in P$ an arbitrary element. Assume the following assumption:

(A1) For any $p \in P$ and $i=1,2, \ldots, k$, there exists $x(i, p) \in W$ such that $g(x(i, p), p) \leq 0$ and $f_{i}(x(i, p)) \leq f_{i}(x)$ for every $x \in W$ with $g(x, p) \leq 0$.

Assumption (A1) just means that the scalar problem

$$
\operatorname{Min}\left\{f_{i}(x): x \in W, g(x, p) \leq 0\right\}
$$

attains an optimal value at $x(i, p) \in W(i=1,2, \ldots, k)$ and, therefore, the ideal point (or the set of partial minima)

$$
J(p)=\left[f_{1}(x(1 ; p)), f_{2}(x(2, p)), \ldots, f_{k}(x(k, p))\right] \in \mathbf{R}^{k}
$$

does exist. In order to achieve an easier notation, we will denote $J_{i}(p)=$ $f_{i}(x(i, p)), i=1,2, \ldots, k$.

Following the approach of [8] or [9], an element $b \in \mathbb{R}^{k}, b \geq 0$, is said to be a balance point of (1) if $\{f(x): x \in W, g(x, p) \leq 0\} \cap[J(p), J(p)+b] \neq \emptyset$ and $\{f(x): x \in W, g(x, p) \leq 0\} \cap\left[J(p), J(p)+b^{*}\right]=\emptyset$ for every $b^{*} \in \mathbb{R}^{k}$ such that $0 \leq b^{*} \leq b, b^{*} \neq b{ }^{6}$ As pointed out in $[5], b \in \mathbb{R}^{k}$ is a balance point of (1) if and only if $J(p)+b$ belongs to the efficient line of (1)

In order to guarantee the existence of balance points in the direction of preferential deviations, we also impose the following assumption:

(A2) For every $p \in P$ there exists a balance point proportional to $d$.

\section{The Envelope Theorem}

Let us introduce the following scalar problem whose decision variables are $\tau \in \mathbb{R}$ and $x \in W$

$$
\operatorname{Min} \tau \quad\left\{\begin{array}{l}
x \in W \\
g(x, p) \leq 0 \\
f(x)-\tau d \leq J(p)
\end{array}\right.
$$

Assumptions $(A 1)$ and $(A 2)$ allows us to establish the statement below

${ }^{6}$ If $u, v \in \mathbf{R}^{k}$ with $u \leq v$, then $[u, v]$ denotes the set $\left\{x \in \mathbf{R}^{k}: u \leq x \leq v\right\}$.
Theorem 1 Assume that $p \in P$ and $\tau_{p} \geq 0$. Then, $\tau_{p} d$ is a balance point of Problem (1) if and only if there exists $x(p) \in W$ such that $\left(x(p), \tau_{p}\right)$ solves (3). ${ }^{7}$ In the affirmative case, $x(p) \in W$ is a Pareto solution of (1) such that $f(x(p))=J(p)+\tau_{p} d .^{8}$

Proof. Suppose that $\tau_{p} d$ is a balance point of (1). Then $J(p)+\tau_{p} d$ is an efficient point and there exists $x(p) \in W$, Pareto solution of (1), such that $f(x(p))=J(p)+\tau_{p} d$. Thus, $f(x(p))-\tau_{p} d=J(p)$ and $\left(x(p), \tau_{p}\right)$ verifies the constraints of (3). Moreover, if $\left(x(p), \tau_{p}\right)$ does not solve (3) we have that there exists a feasible couple $(x, \tau)$ such that $\tau<\tau_{p}$. Since $(x, \tau)$ is feasible we have that $f(x)-J(p) \leq \tau d<\tau_{p} d$. Hence $f(x) \in[J(p), J(p)+\tau d]$ and this is a contradiction because $\tau_{p} d$ is a balance point of $(1)$ and $\tau<\tau_{p}$.

Conversely, let us suppose that $\left(x(p), \tau_{p}\right)$ solves (3) but $\tau_{p} d$ is not a balance point of (1). (A2) guarantees the existence of a balance point $\tau^{*} d$. Assume at the moment that $\tau^{*}>\tau_{p}$. Since $\left(x(p), \tau_{p}\right)$ solves (3) it must be (3)-feasible and, therefore, $f(x(p))-J(p) \leq \tau_{p} d<\tau^{*} d$. Thus $f(x(p)) \in\left[J(p), J(p)+\tau_{p} d\right]$ and this is a contradiction because $\tau^{*} d$ is a balance point. Assume now that $\tau^{*}<\tau_{p}$. Since $\tau^{*} d$ is a balance point there exists $x \in W$ such that $x$ is a Pareto solution of $(1)$ and $J(p)+\tau^{*} d=f(x)$. Therefore, $\left(x, \tau^{*}\right)$ is (3)-feasible and $\tau^{*}<\tau_{p}$ which contradicts the hypothesis because $\tau_{p}$ must be the minimum value of (3).

The latter theorem allows us to compute the balance point associated with the direction of preferential deviations. In fact, in a first step the ideal point $J(p)$ may be computed by solving $k$ scalar problems and, later, once $J(p)$ is known, Problem (3) leads to the balance point $\tau_{p} d$.

As a consequence, the sensitivity of Problem (1) with respect to the parameter $p$ depends on the sensitivity of $k+1$ scalar problems. Theorem 2 establishes this property with precision and extends the classical envelope theorem, well-known in the scalar programming case.

Theorem 2 Let us assume that $W$ and $P$ are open sets and $f$ and $g$ are continuously differentiable functions. Denote by

$$
\lambda(i, p)=\left(\lambda_{1}(i, p), \lambda_{2}(i, p), \ldots, \lambda_{m}(i, p)\right) \in \mathbb{R}^{m}
$$

the Kuhn-Tucker multiplier of (2) associated to $x(i, p)(i=1,2, \ldots, k, p \in$ P). Suppose that $(\mu(p), \nu(p))=\left(\mu_{1}(p), \ldots, \mu_{m}(p), \nu_{1}(p), \ldots, \nu_{k}(p)\right) \in \mathbb{R}^{m+k}$ represents the Kuhn-Tucker multiplier of (3) for each $p \in P$. Consider finally that all of the Kuhn-Tucker multipliers continuously depend on $p \in P$ and ${ }^{7}$ If $\tau_{0} d$ is a balance point for Problem $(1)$ then the existence of $x(p)$ may be proved
without imposing Assumption $(A 2)$.
${ }^{8}(A 2)$ and Theorem 1 guarantee the existence of solutions of $(3)$. Henceforth $\tau_{p} \geq 0$
will represent the optimal value of $(3)$, and $x(p)$ will represent the corresponding Pareto
solution of $(1)$. 
define the function $P \ni p \longrightarrow F(p)=J(p)+\tau_{p} d=f(x(p))$. Then,

$$
\begin{aligned}
\frac{\partial F_{r}}{\partial p_{s}}= & \sum_{j=1}^{m} \lambda_{j}(r, p) \frac{\partial g_{j}}{\partial p_{s}}(x(r, p), p) \\
& +d_{r} \sum_{j=1}^{m} \mu_{j}(p) \frac{\partial g_{j}}{\partial p_{s}}(x(p), p) \\
& -d_{r} \sum_{i=1}^{k} \sum_{j=1}^{m} \nu_{i}(p) \lambda_{j}(i, p) \frac{\partial g_{j}}{\partial p_{s}}(x(i, p), p)
\end{aligned}
$$

holds for $r=1,2, \ldots, k, s=1,2, \ldots, l$ and every $p \in P{ }^{9}$

Proof. The envelope theorem of scalar programming guarantees that

$$
\frac{\partial J_{r}}{\partial p_{s}}=\sum_{j=1}^{m} \lambda_{j}(r, p) \frac{\partial g_{j}}{\partial p_{s}}(x(r, p), p)
$$

Thus, the proof will be completed if we show that the function $P \ni p \longrightarrow$ $h(p)=\tau_{p} \in \mathbb{R}$ verifies the equality

$$
\frac{\partial h}{\partial p_{s}}=\sum_{j=1}^{m} \mu_{j}(p) \frac{\partial g_{j}}{\partial p_{s}}(x(p), p)-\sum_{i=1}^{k} \nu_{i}(p)\left[\sum_{j=1}^{m} \lambda_{j}(i, p) \frac{\partial g_{j}}{\partial p_{s}}(x(i, p), p)\right]
$$

The Lagrangian function of (3) is given by

$$
L(\tau, x, p, \mu, \nu)=\tau+\sum_{j=1}^{m}\left[\mu_{j} g_{j}(x, p)\right]+\sum_{i=1}^{k} \nu_{i}\left[f_{i}(x)-\tau d_{i}-J_{i}(p)\right]
$$

being $\mu=\left(\mu_{1}, \mu_{2}, \ldots, \mu_{m}\right) \in \mathbb{R}^{m}$ and $\nu=\left(\nu_{1}, \nu_{2}, \ldots, \nu_{k}\right) \in \mathbb{R}^{k}$. Therefore, the envelope theorem of scalar programming ensures that

$$
\frac{\partial h}{\partial p_{s}}=\frac{\partial L}{\partial p_{s}}=\sum_{j=1}^{m}\left[\mu_{j} \frac{\partial g_{j}}{\partial p_{s}}(x(p), p)\right]-\sum_{i=1}^{k} \nu_{i}\left[\frac{\partial J_{i}}{\partial p_{s}}\right]
$$

and (6) trivially follows from (5).

The latter theorem can be particularized in order to obtain the sensitivity of $F$ with respect to the term of the right side. In this case (4) may be significantly simplified.

\footnotetext{
${ }^{9}$ Let us remark that the partial derivative $\frac{\partial g_{j}}{\partial p_{s}}$ is evaluated at $k+1$ different points
}

Corollary 3 Assume that $l=m$ and $g(x, p)=\bar{g}(x)-p$, being $\bar{g}: W \longrightarrow$ $\mathbb{R}^{m}$ an arbitrary function. Then, under the assumptions of Theorem 2 , the following expression

$$
\frac{\partial F_{r}}{\partial p_{s}}=-\left[\lambda_{s}(r, p)+\mu_{s}(p) d_{r}-d_{r} \sum_{i=1}^{k} \nu_{i}(p) \lambda_{s}(i, p)\right]
$$

holds for $r=1,2, \ldots, k, s=1,2, \ldots, m$ and every $p \in P$.

\section{Convex Problems and Duality Theory}

Throughout this section we will assume that $W$ is a convex set, $f_{i}$ is a convex function $(i=1,2, \ldots, k), l=m$ and $g(x, p)=\bar{g}(x)-p$ being $\bar{g}: W \longrightarrow \mathbb{R}^{m}$ an arbitrary convex function (i.e., its components $\bar{g}_{j}: W \longrightarrow \mathbb{R}, j=1,2, \ldots, m$, are convex functions). Therefore, (1) is a convex vector problem and (2) and (3) are convex scalar problems.

Consider an arbitrary matrix $\Lambda=\left(\lambda_{i, j}\right)_{i=1,2, \ldots, k}^{j=1,2, \ldots, m}$ and two elements $\mu=$ $\left(\mu_{1}, \ldots, \mu_{m}\right) \in \mathbb{R}^{m}$ and $\nu=\left(\nu_{1}, \ldots, \nu_{k}\right) \in \mathbb{R}^{k}$. Suppose that all the components of these matrices are nonnegative. Then, $(\Lambda, \mu, \nu)$ is said to be dualfeasible if the sets $\left\{f(x)+(\bar{g}(x)-p) \Lambda^{T}: x \in W\right\}$ and $\left\{\tau+(\bar{g}(x)-p) \mu^{T}+\right.$ $\left.(f(x)-\tau d-J(p)) \nu^{T}: x \in W, \tau \in \mathbb{R}\right\}$ are bounded from below in $\mathbf{R}^{k}$ and $\mathbf{R}$; respectively, ${ }^{10}$ in which case we will define the dual objectives by ${ }^{11}$

$$
\varphi(\Lambda)=\operatorname{Inf}\left\{f(x)+(\bar{g}(x)-p) \Lambda^{T}: x \in W\right\} \in \mathbb{R}^{k}
$$

and

$\psi(\mu, \nu)=\operatorname{Inf}\left\{\tau+(\bar{g}(x)-p) \mu^{T}+(f(x)-\tau d-J(p)) \nu^{T}: x \in W, \tau \in \mathbb{R}\right\} \in \mathbb{R}$

The dual problem of (1) is given by

$$
\operatorname{Max}(\varphi(\Lambda), \psi(\mu, \nu))\{(\Lambda, \mu, \nu) \in \Gamma
$$

being $\Gamma$ the set of dual-feasible elements. ${ }^{12}$

It is clear that (10) is a vector problem with $k+1$ objectives, but there exists a simple relationship between the primal and the dual objectives.

Lemma 4 If $(\Lambda, \mu, \nu) \in \Gamma$, then the following assertions are fulfilled: a) $\varphi(\Lambda) \leq J(p)$ and $\psi(\mu, \nu) \leq \tau_{p}$

b) If $\bar{x} \in W$ verifies $\bar{g}(\bar{x}) \leq p$, then expressions $f(\bar{x}) \leq \varphi(\Lambda)+\psi(\mu, \nu) d$ and $f(\bar{x}) \neq \varphi(\Lambda)+\psi(\mu, \nu) d$ cannot simultaneously hold.

${ }^{10}$ As usual, if $M$ is an arbitrary matrix, $M^{T}$ will denote the transpose matrix.
${ }^{11}$ Recall that $\mathrm{R}^{k}$ is an order complete Banach lattice (

${ }^{11}$ Recall that $\mathrm{R}^{k}$ is an order complete Banach lattice (see, for instance, [14]) and, con8) and (9) expressions of previous literature when dealing with dual 12Nontico 
Proof. Since $\lambda_{i, j} \geq 0$ for $i=1,2, \ldots, k$ and $j=1,2, \ldots, m$, it is obvious that $\varphi(\Lambda) \leq \operatorname{In} f\left\{f(x)+(\bar{g}(x)-p) \Lambda^{T}: x \in W, \bar{g}(x)-p \leq 0\right\} \leq \operatorname{Inf}\{f(x)$ $x \in W, \bar{g}(x)-p \leq 0\}=J(p)$.

Analogously, $\psi(\mu, \nu) \leq \operatorname{Inf}\left\{\tau+(\bar{g}(x)-p) \mu^{T}+(f(x)-\tau d-J(p)) \nu^{T}\right.$ $x \in W, \bar{g}(x)-p \leq 0, \tau \in \mathbb{R}, f(x)-\tau d-J(p) \leq 0\} \leq$ Inf $\{\tau: x \in W$ $\bar{g}(x)-p \leq 0, \tau \in \mathbb{R}, f(x)-\tau d-J(p) \leq 0\}=\tau_{p}$

Moreover, bearing in mind $a$ ), if $f(\bar{x}) \leq \varphi(\Lambda)+\psi(\mu, \nu) d$ then $f(\bar{x}) \leq$ $J(p)+\tau_{p} d=f(x(p))$. Thus, $f(\bar{x})=f(x(p))$ since $x(p)$ is a Pareto solution of (1).

Hereafter the following Slater qualification is imposed:

(A3) The vector of preferential deviations satisfies the inequality $d_{i}>0$ for $i=1,2, \ldots, k$, and for any $p \in P$ there exists $x^{p} \in W$ such that $\bar{g}_{j}\left(x^{p}\right)-p_{j}<0$, $j=1,2, \ldots, m$.

As will immediately be shown, the Slater qualification guarantees the existence of strong dual solutions and the absence of duality gap.

Theorem 5 Given an arbitrary $p \in P$, there exists $(\Lambda(p), \mu(p), \nu(p)) \in \Gamma$ such that:

a) $\varphi(\Lambda(p)) \geq \varphi(\Lambda)$ and $\psi(\mu(p), \nu(p)) \geq \psi(\mu, \nu)$ for every $(\Lambda, \mu, \nu) \in \Gamma{ }^{13}$

b) $\varphi(\Lambda(p))=J(p), \psi(\mu(p), \nu(p))=\tau_{p}$, and $\varphi(\Lambda(p))+\psi(\mu(p), \nu(p)) d$ $=f(x(p))$

Proof. Assertion $a$ ) trivially follows from $b$ ) and Lemma 4, so let us prove $b)$. Let $i \in\{1,2, \ldots, k\}$ and consider the convex and scalar Problem (2). The saddle point theorem of [13] (Chapter IX) guarantees the existence of

$$
\lambda_{i}(p)=\left(\lambda_{i, 1}(p), \lambda_{i, 2}(p), \ldots, \lambda_{i, m}(p)\right)
$$

such that $\lambda_{i, j}(p) \geq 0, j=1,2, \ldots, m$, and

$$
\operatorname{Inf}\left\{f_{i}(x)+(\bar{g}(x)-p) \lambda_{i}(p)^{T}: x \in W\right\} \geq J_{i}(p)
$$

Now, it is clear that $\Lambda(p)=\left(\lambda_{i, j}(p)\right)_{i=1,2, \ldots, k}^{j=1,2, \ldots, m}$ satisfies the required condition. Furthermore, since (3) is also a scalar convex problem that verifies the Slater qualification, analogous arguments lead to the existence of $\mu(p)$ and $\nu(p)$.

The absence of duality gap and the existence of strong dual solutions allow us to characterize dual solutions and balance points by means of saddle points and complementary slackness conditions. Furthermore, the saddle point condition is stated by means of a vector inequality instead of the failure to hold the opposite inequality, as usual in the vector programming case

\footnotetext{
${ }^{13}$ i.e., $(\Lambda(p), \mu(p), \nu(p)) \in \Gamma$ is a strong solution of (10)
}

Theorem 6 Consider an arbitrary (3)-feasible $(\bar{x}, \bar{\tau}) \in W \times \mathbf{R}$ and $(\Lambda, \mu, \nu)$ $\in \Gamma$. Then $(\bar{x}, \bar{\tau})$ solves (3) ${ }^{14}$ and $(\Lambda, \mu, \nu)$ solves (10) if and only if

$$
f(\bar{x})+(\bar{g}(\bar{x})-p) \Lambda^{T} \leq f(x)+(\bar{g}(x)-p) \Lambda^{T}
$$

for every $x \in W$,

$$
\begin{aligned}
& \bar{\tau}+(\bar{g}(\bar{x})-p) \mu^{T}+(f(\bar{x})-\bar{\tau} d-\varphi(\Lambda)) \nu^{T} \\
\leq \quad & \tau+(\bar{g}(x)-p) \mu^{T}+(f(x)-\tau d-\varphi(\Lambda)) \nu^{T}
\end{aligned}
$$

for every $x \in W$ and every $\tau \in \mathbb{R}$, and the complementary slackness conditions

$$
\begin{aligned}
& (\bar{g}(\bar{x})-p) \Lambda^{T}=0 \\
& (\bar{g}(\bar{x})-p) \mu^{T}=0
\end{aligned}
$$

and

$$
(f(\bar{x})-\bar{\tau} d-\varphi(\Lambda)) \nu^{T}=0
$$

hold.

Proof. Suppose that $(\bar{x}, \bar{\tau})$ solves $(3)$ and $(\Lambda, \mu, \nu)$ solves (10). Theorem 1 guarantees that $\bar{x}$ solves (1) and, therefore, the results of [13] (Chapter $I X)$ apply on Problems (2) $(i=1,2, \ldots, k)$ and (3) and show that (11), (13) and (14) hold and (12) and (15) also hold if $\varphi(\Lambda)$ is substituted by $J(p)$. Hence, the conclusion trivially follows from the equality $\varphi(\Lambda)=J(p)$ already established in the previous theorem.

Conversely, assume (11), (12) (13), (14) and (15). Then, (8) and (11) show that $\varphi(\Lambda)=f(\bar{x})+(\bar{g}(\bar{x})-p) \Lambda^{T}$ and, consequently, (13) leads to $\varphi(\Lambda)=f(\bar{x}) \geq J(p)$. Now, Lemma 4 proves that $\varphi(\Lambda)=J(p)$. Analogous arguments lead to $\psi(\mu, \nu)=\tau_{p}$ and the latter theorem proves that $(\Lambda, \mu, \nu)$ is a dual solution. Moreover, $\varphi(\Lambda)=J(p)$ implies that (12) and (15) hold if $\varphi(\Lambda)$ is substituted by $J(p)$, and these conditions, along with (14) and the results of [13], permit us to ensure that $(\bar{x}, \bar{\tau})$ solves (3). Once the complementary slackness conditions have been provided, standard arguments permit us to establish the equivalence between dual solutions and Lagrange multipliers when dealing with convex and differentiable problems. Thus, Theorem 2 and Corollary 3 may be adapted so that one can measure the primal sensitivity by means of dual solutions. For instance, the following result, whose proof is omitted, may be easily obtained.

Corollary 7 Let $P$ be an open (and convex) set. Denote by $(\Lambda(p), \mu(p), \nu(p))$ $\in \Gamma$ the strong dual solution of (10) (for every $p \in P$ ) whose existence is guaranteed by Theorem 5. Suppose that the function $P \ni p \longrightarrow(\Lambda(p), \mu(p), \nu(p))$

${ }^{14}$ Recall that Theorem 1 ensures that $(\bar{x}, \bar{\tau})$ solves (3) if and only if $\bar{x}$ is a Pareto solution of (1) and $\bar{\tau} d$ is a balance point such that $J(p)+\bar{\tau} d=f(\bar{x})$. 
is continuous. Then, the function $P \ni p \longrightarrow F(p)=\varphi(\Lambda(p))+\psi(\mu(p), \nu(p)) d \in$ $\mathbb{R}^{k}$ is continuously differentiable and

$$
\frac{\partial F_{r}}{\partial p_{s}}=-\left[\lambda_{r, s}(p)+\mu_{s}(p) d_{r}-d_{r} \sum_{i=1}^{k} \nu_{i}(p) \lambda_{i, s}(p)\right]
$$

for $r=1,2, \ldots, k, s=1,2, \ldots, m$ and every $p \in P$.

Remark 8 Notice that (3) is linear if (1) is linear, in which case it may be proved that (10) is also linear because $\Gamma$ may be given by linear constraints and $\varphi$ and $\psi$ have a linear expression (see [1] or [3] for further details on this point in a very general setting). Hence, in the important linear case, the balance space approach yields a linear dual problem with a strong solution that avoids the duality gap, characterizes the balance points by means of complementary slackness conditions (no saddle point conditions are required in this case) and measures the primal sensitivity with respect to any parameter appeared in the problem. These properties are identical to those observed in the scalar programming and usually fail when dealing with classical duals in vector optimization.

\section{Conclusions}

This paper has shown how the theory of global optimization and the balance space approach may apply in order to develope a general theory of duality and sensitivity for vector optimization problems. This general theory points out that the balance space approach is an interesting alternative and complements the classical Pareto approach. The theory overcomes several caveats usual in the literature. For instance, it yields a general envelope theorem that easily applies in practical situations and measures the sensitivity with respect to any parameter of the problem. Regarding the duality theory for convex (and linear) problems, the balance set and the set of dual solutions may be characterized by means of saddle point conditions whose statements may be given in terms of vector inequalities. Consequently, our saddle point conditions seem to be more effective than those obtained by previous literature, and they lead, along with some complementary slackness conditions, to a system of equations providing us with the efficient line, the set of dual solutions and the sensitivity with respect to any parameter

\section{References}

[1] Balbás, A., Ballvé, M. and P.J. Guerra, 1999, "Sensitivity analysis in multiobjective programming under homogeneity assumptions." Journal of Multi-Criteria Decision Analysis, 8, 133-138.
[2] Balbás, A., Fernández, F.J. and P.J. Guerra, 1995, "On the envolven theorem in multiobjective programming." Indian Journal of Pure and Applied Mathematics, 26, 11, 1035-1047.

[3] Balbás, A. and P.J. Guerra, 1996, "Sensitivity analysis for convex multiobjective programming in abstract spaces." Journal of Mathematical Analysis and Applications, 202, 645-658.

[4] Balbás, A. and A. Heras, 1993, "Duality theory for infinite-dimensional multiobjective linear programming." European Journal of Operational Research, 68, 379-388.

[5] Ehrgott, M., Hamacher, H.W., Klamroth, K., Nickel, S., Schobel, A and M.M. Wiecek, 1997, "A Note on the equivalence of balance points and Pareto solutions in multiple objective programming." Journal of Optimization. Theory and Applications, 92, 209-212.

[6] Galperin, E.A., 1990, "The Cubic Algorithm for Optimization and Control." NP Research Publication. Montreal, Québec, Canada.

[7] Galperin, E.A., 1992, "Nonscalarized multiobjective global optimization." Journal of Optimization. Theory and Applications, 75, 69-85.

[8] Galperin, E.A., 1997, "Pareto analysis vis-à-vis balance space approach in multiobjective global optimization," Journal of Optimization. Theory and Applications, 93, 3, 533-545.

[9] Galperin, E.A. and M.M. Wiecek, 1999, "Retrieval and use of the balance set in multiobjective global optimization," Computers and Mathematics with Applications, 37, 111- 123

[10] Jahn, J., 1986, "Mathematical vector optimization in partially ordered linear spaces." Verlag Peter Lang, Frankfurt am Main.

[11] Klose, J., 1992, "Sensitivity analysis using the tangent derivative." $N_{u-}$ merical Functional Analysis and Optimization, 13, 143-153.

[12] Kuk, H., Tanino, J. and M. Tanaka, 1996, "Sensitivity analysis in vector optimization. "Journal of Optimization. Theory and Applications, 89 713-730.

[13] Luenberger, D.G., 1969, "Optimization by vector space methods." Wiley. New York.

[14] Meyer-Nieberg, P., 1991, "Banach lattices." Springer-Verlag. New York.

[15] Sawaragi, Y., H. Nakayama and T. Tanino, 1985, "Theory of multiobjective optimization." Academic Press. 
[16] Zowe, S., 1977, "The saddle point theorem of Kuhn and Tucker in ordered vector spaces." Journal of Mathematical Analysis and Applications, 57, 41-55. 